\title{
Archéopages
}

Archéopages

Archéologie et société

Hors-série 2 | 2010

Archéologie sans frontières

\section{Église Gabriel, corpus céramologique}

\section{Kristell Chuniaud}

\section{OpenEdition}

\section{Journals}

Édition électronique

URL : https://journals.openedition.org/archeopages/760

DOI : 10.4000/archeopages.760

ISSN : 2269-9872

\section{Éditeur}

INRAP - Institut national de recherches archéologiques préventives

\section{Édition imprimée}

Date de publication : 1 octobre 2010

Pagination : $43-45$

ISSN : 1622-8545

\section{Référence électronique}

Kristell Chuniaud, «Église Gabriel, corpus céramologique », Archéopages [En ligne], Hors-série 2 | 2010, mis en ligne le 01 octobre 2010, consulté le 23 février 2023. URL : http://journals.openedition.org/ archeopages/760 ; DOI : https://doi.org/10.4000/archeopages.760 
rites lors du déroulement des cérémonies? S'agit-il d'un mobilier spécialisé, consacré exclusivement aux coutumes funéraires, ou au contraire d'un vaisselier domestique reconverti au monde des morts? Les multiples interrogations sur les pratiques locales sont d'autant plus délicates à résoudre que notre perception des phénomènes peut être tronquée par la disparition éventuelle de contenants en matériaux périssables (vanneries, calebasses); de plus, l'état de la recherche archéologique ne permet pas de comparer cet ensemble à des lots trouvés sur des sites d'habitat ou des espaces cultuels. Létude du lot de Tater Gur révèle néanmoins quelques similitudes troublantes, en termes typologiques et décoratifs, avec le faciès connu dans le Tigré après la disparition du royaume d'Axoum, vers les vinI ${ }^{\mathrm{e}}$-IX ${ }^{\mathrm{e}}$ siècles (Grey/Black Aksumite Ware). Sur le continent africain, la céramique passe pour un bien courant, qui fait rarement l'objet d'un trafic à longue distance, car non suffisamment rentable. Aussi, est-il probable que les fabrications de Tater Gur et des sites postaxoumites correspondent à des séries distinctes, issues d'ateliers autonomes bien que puisant leur inspiration dans un fonds culturel commun.

La vaisselle découverte dans le tumulus pourrait en effet s'inscrire, non pas dans une démarche commerciale, mais plutôt dans un processus d'acculturation dont il conviendrait de définir les modalités. Au demeurant, la forme et les aspects ornementaux de certaines productions de l'époque axoumite classique (Red et Brown Aksumite Ware) suggèrent que le répertoire du Haut-Moyen Âge pourrait plonger ses racines dans la tradition céramique du royaume chrétien.

D'autres travaux seraient toutefois nécessaires pour valider ou non cette proposition, puisque ces problèmes revêtent de fortes implications historiques.

\section{L'église Gabriel, le plan}

Anne-Marie Jouquand
Inrap
Deresse Ayenachew
Authority for Research and Conservation of Cultural Heritage
Régis Bernard
Inrap
Bernard Farago-Szekeres
Inrap

Léglise Gabriel occupe le sommet d'une éminence rocheuse d'une dizaine de mètres de hauteur et de $350 \mathrm{~m}^{2}$. Le plan général des vestiges, bien qu'incomplet, évoque le modèle basilical des églises éthiopiennes : un bâtiment rectangulaire maçonné, de 7,40 m sur 10,50 m, composé de deux pièces (a et b) et orienté ouest-est [Fig. 1]. Une galerie (c) court le long du bâtiment central, ménageant un espace pour desservir les trois entrées de l'église. La construction de cet ensemble de $186 \mathrm{~m}^{2}$ semble avoir été réalisée d'un seul jet. On reconnaît traditionnellement à l'est (a), le maqdas, le saint des saints, dont l'accès est réservé aux prêtes et à l'ouest (b), la nef d'où les fidèles suivaient l'office. Fait troublant, aucun pilier ne ponctue cet espace, contrairement à la plupart des églises éthiopiennes.
L'accès à l'église s'effectuait en contrebas (d), depuis l'ouest, par un aménagement en bois (échelle ou escalier) encastré dans le rocher. Chaîné aux massifs encadrant l'entrée, un muret ceinture l'ensemble.

Trois temps ponctuent l'histoire de ce lieu [Fig.2]. Deux corps d'adultes déposés dans une fosse (F10) et quelques pièces lithiques témoignent d'une fréquentation ancienne de l'amba. L'église Gabriel est construite, probablement au XV ${ }^{\mathrm{e}}$ siècle, après des travaux de nivellement. Cinq tombes, appartenant sans doute à des personnages de haut rang, sont implantées à l'intérieur de l'édifice [Fig.3]. Quels liens entretenait la modeste église Gabriel avec l'église royale toute proche établie à Meshala Maryam? On ne saurait le dire, mais après un violent incendie, une petite centaine de tombes prend place sur les ruines de l'édifice. La présence d'un petit cimetière tardif illustre ici de façon saisissante la permanence d'une tradition chrétienne attachée à l'amba : une petite communauté va venir ensevelir ses morts à l'emplacement d'une église détruite et désacralisée. L'attraction du lieu demeure donc très forte et pourrait être en lien avec les inhumations privilégiées établies au cœur du sanctuaire.

\section{Église Gabriel, corpus céramologique \\ Kristell Chuniaud \\ Inrap}

Létude a porté sur les céramiques de deux sites médiévaux des hauts plateaux éthiopiens (région du nord du Choa) dont la nature diffère radicalement : un lieu à vocation cultuelle et funéraire - une église - sur l'amba Gabriel, et le site de Meshala Maryam, identifié d'après la tradition orale comme le siège d'un camp royal. Outre la définition (technique, morphologie, approche des fonctions) des poteries en présence, l'enjeu était d'identifier d'éventuelles variations dans les faciès céramiques des deux sites et de les interpréter en termes de décalage chronologique ou de différence dans la nature des occupations. En raison du caractère pionnier des recherches archéologiques dans la région considérée et de l'absence de références antérieures dans le domaine céramologique, l'étude s'est effectuée seulement à partir du matériau brut et inédit que constituent les collections recueillies sur le terrain. Les poteries ont été abordées d'un point de vue purement descriptif et morphologique, sans aucune connaissance préalable de la datation précise des sites au sein de la période du Moyen Âge, ni de l'éventuel décalage chronologique pouvant exister entre eux.

L'étude des céramiques de l'amba Gabriel a été privilégiée car le site se prête davantage à l'établissement d'un corpus de référence. Du fait de son caractère sacré et funéraire, et peut-être aussi en raison de son isolement topographique, l'amba Gabriel n'a pas connu un changement radical de vocation après son abandon. Même s'il est impossible d'exclure une fréquentation du site jusqu'à nos jours, la dernière période ne comprend 

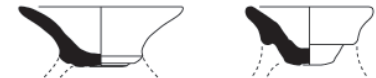

1

1

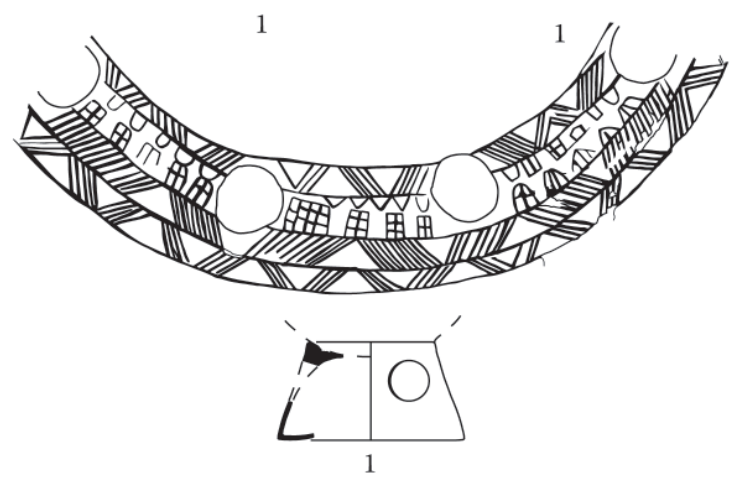

$\overbrace{4}^{\infty}$
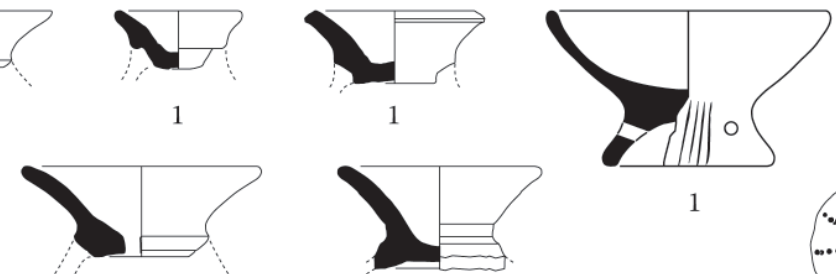

1
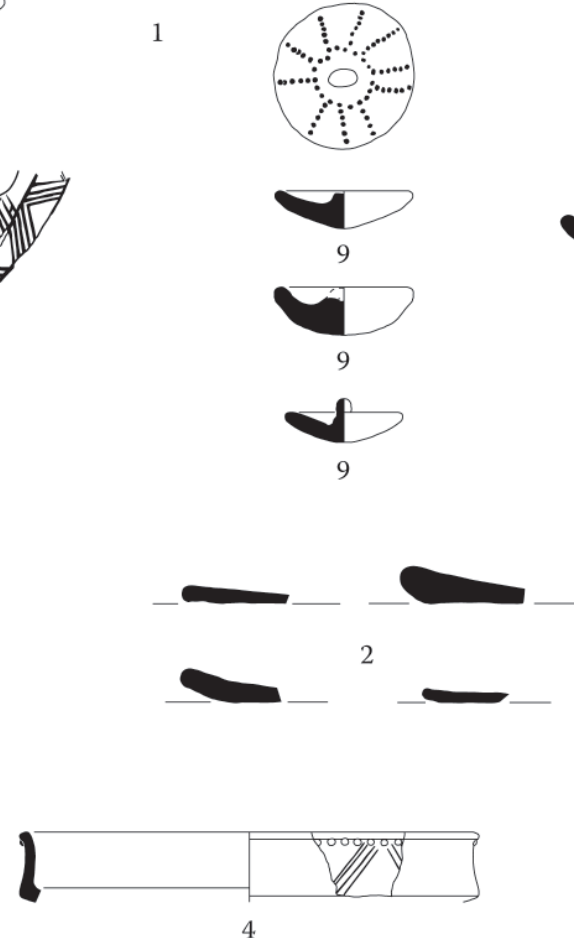

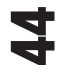
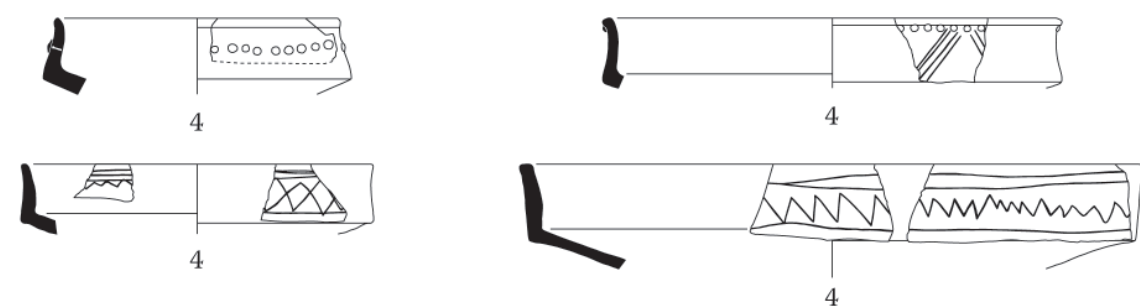

)

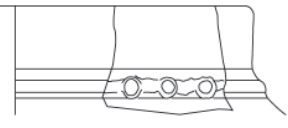

3
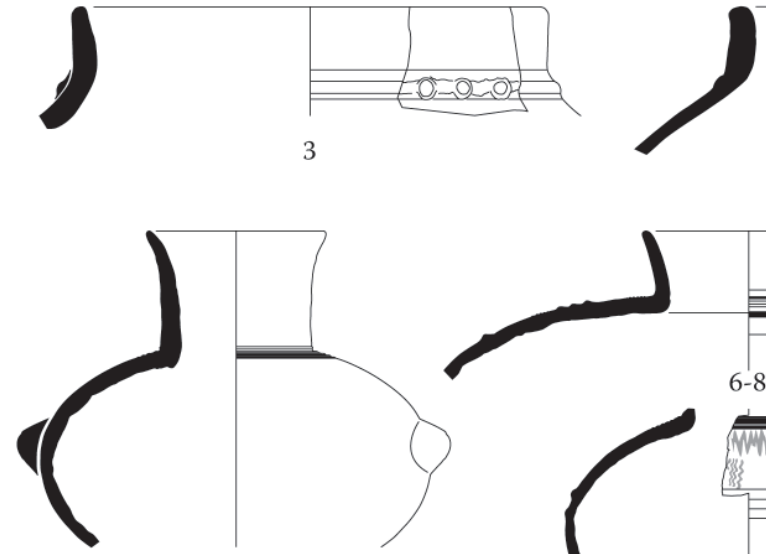

6-8

[Fig.1] Planche typologique raisonnée du corpus céramologique du site de l'Église Gabriel.

\section{(1)}

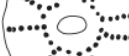

$\because:$
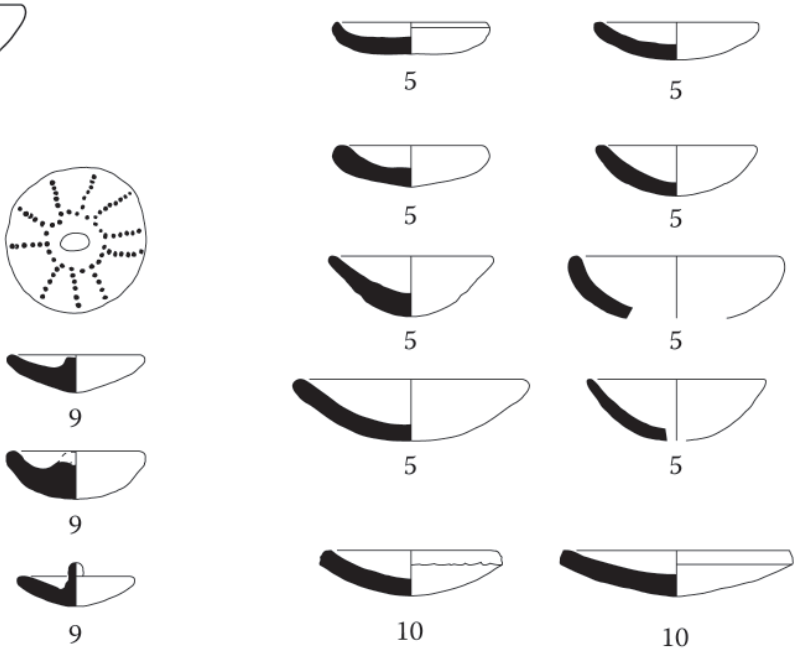

10
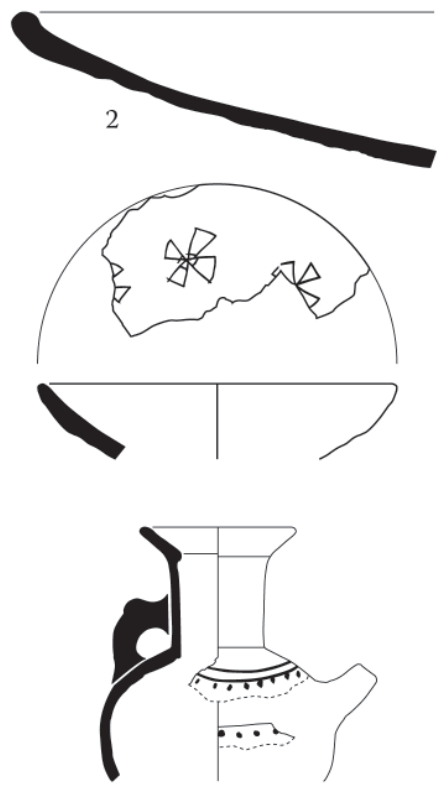

3
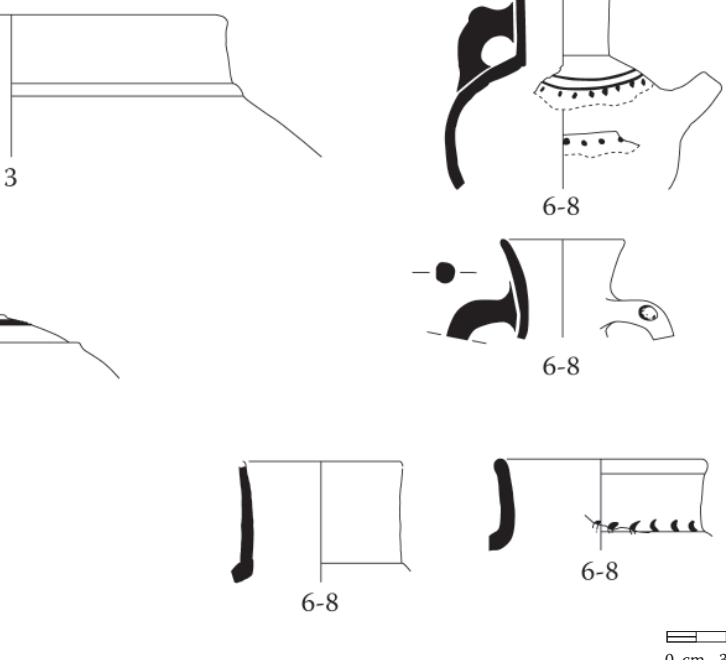
pas de réoccupation majeure de nature résidentielle par exemple. Le mobilier recueilli est donc susceptible de fournir une image assez juste des céramiques utilisées dans l'église pendant ses différentes périodes d'occupation. Le corpus ainsi obtenu pourra servir de base de comparaison pour des recherches futures dans cette région, en vue notamment d'établir des parallèles chronologiques. À titre d'hypothèse de travail, une distinction a été faite entre les récipients qui pourraient avoir un lien étroit avec les activités spécifiques pratiquées dans un lieu de culte et les autres formes qui paraissent relever d'une utilisation domestique de la céramique. [Fig.1] Aux premiers répondent les coupelles de petite taille, compactes et massives (formes 1,5 et 10), en lien vraisemblable avec l'utilisation de l'encens. La forme 1 présente d'ailleurs une stricte analogie morphologique avec les coupelles à encens contemporaines. Les secondes semblent en lien avec l'emploi de denrées consommables : cuisson des aliments ( « plats à inger ${ }^{7}$ » de forme 2), stockage des denrées (forme 3), préparation et présentation des aliments (coupes carénées de forme 4 et autres plats), approvisionnement et stockage de l'eau (pots à eau de forme 7), service des liquides avec les cruches et/ou vases de forme 6-8, voire préparation de laitages avec des panses perforées assimilables à des faisselles.

Par ailleurs, l'examen des céramiques recueillies dans un contexte particulier de l'édifice (fosse F1) permet de poser l'hypothèse d'un mobilier liturgique prestigieux, dont l'usage est réservé aux prêtres, par la présence de céramique importée (Céladon de Chine) et de vases a priori antérieurs à la phase d'occupation de l'église, qui trouvent des parallèles morphologiques auprès des céramiques de la culture Shay, illustrée dans la présente livraison par les vases du tumulus de Tater Gur (cf. encadré p. 4.1). Ces vases plus anciens pourraient devoir leur conservation à la nature sacrée du lieu.

Les tentatives de mise en parallèle des mobiliers céramiques des deux sites tendent à exclure, pour le site de Meshala Maryam, toute occupation « royale » des lieux telle que la tradition orale pouvait le laisser envisager. Les vases présents témoignent d'une occupation humaine de nature domestique. Cette caractéristique, associée à l'absence de mobilier de prestige ou importé, ne permet pas de déceler sur ce site l'empreinte d'un roi et de sa cour, fût-elle itinérante comme en ces temps médiévaux.

Ce travail est à considérer comme un premier jalon dans la connaissance de la culture matérielle des sites médiévaux des hauts plateaux éthiopiens.
L'approche géomorphologique du site de Lalibela

$$
\begin{aligned}
& \text { Laurent Bruxelles } \\
& \text { Inrap }
\end{aligned}
$$

On peut se demander, a priori, ce que pourrait apporter un géomorphologue dans l'étude d'églises, fussent-elles creusées dans le roc. Mais rapidement, plusieurs faits ont été observés qui ont paru contribuer à la compréhension du site.

Tout d'abord, la forme des églises est directement conditionnée par la topographie initiale du secteur. Celles du groupe 1 sont alignées avec des toits à double pentes ; elles ont été creusées dans un relief particulièrement propice puisque la crête était orientée est-ouest. La ligne d'interfluve correspond peu ou prou au faîtage des églises. En revanche, dans le groupe 3, Beta Giyorgis a un toit plan, légèrement incliné, conformément à la pente du versant dans lequel il a été entaillé. Ensuite, le creusement dans ce site a bénéficié des scories basaltiques qui se prêtent particulièrement bien à la taille. Les parties les plus tendres de la roche ont été exploitées pendant les premières phases d'occupation du site (troglodytique et hypogées). Les phases ultérieures ont recoupé une roche basaltique beaucoup plus résistante à la taille, posant manifestement des problèmes techniques.

Enfin, nous avons réalisé un important travail d'identification et de topographie des déblais correspondant au creusement de chaque groupe d'églises. Ces tas, qui peuvent dépasser $20 \mathrm{~m}$ d'épaisseur, recèlent en leur sein une stratigraphie qui n'existe plus au niveau des bâtiments euxmêmes, puisqu'ils sont tous excavés. Nous disposons donc là du meilleur moyen de dater le site, mais aussi de caler les principales phases de creusement de chaque groupe d'églises.
Galette de céréal qui forme la base de l'alimentation. 